\title{
Reversibility Error of Image Interpolation Methods: Definition and Improvements
}

\author{
Thibaud Briand ${ }^{1,2}$ \\ ${ }^{1}$ Université Paris-Est, LIGM (UMR CNRS 8049), ENPC, F-77455 Marne-la-Vallée, France \\ ${ }^{2}$ Université Paris-Saclay, CMLA (UMR CNRS 8536), France \\ (briand.thibaud@gmail.com) \\ Communicated by Jean-Michel Morel and Pascal Monasse \\ Demo edited by Thibaud Briand
}

\begin{abstract}
There is no universal procedure in image processing for evaluating the quality and performance of an interpolation method. In this work, we introduce a new quantity: the reversibility error. For a given image, it measures the error after applying successively a homography close to the identity, a crop (removing boundary artifacts) and the inverse homography. An average over random homographies is made to remove the dependency on the homography. A more precise measurement discarding very high-frequency artifacts is obtained by clipping the spectrum of the difference. We also propose new fine-tuned interpolation methods that are based on the DFT zoom-in and pre-existing (or base) interpolation methods. The zoomed version of an interpolation method is obtained by applying it to the DFT zoom-in of the image. In the periodic plus smooth version of interpolation methods, the non-periodicity is handled by applying the zoomed version to the periodic component and a base interpolation method to the smooth component. In an experimental part, we show that the proposed fine-tuned methods have smaller reversibility errors than their base interpolation methods and that the error is mainly localized in a small high-frequency band. We recommend to use the periodic plus smooth versions of high order B-spline. It is more efficient and provides better results than trigonometric polynomial interpolation.
\end{abstract}

\section{Source Code}

The ANSI C99 implementation of the code that we provide is the one which has been peer reviewed and accepted by IPOL. The source code, the code documentation, and the online demo are accessible at the IPOL web page of this article ${ }^{1}$. Compilation and usage instructions are included in the README.txt file of the archive.

Keywords: interpolation; reversibility error; periodic plus smooth decomposition; B-spline interpolation; trigonometric polynomial interpolation

\footnotetext{
${ }^{1}$ https://doi.org/10.5201/ipol.2019.277
} 


\section{Introduction}

Interpolation is one of the most basic tools of image processing. Indeed, the evaluation of an image at subpixel locations is required in many algorithms. The overall performance of there algorithms therefore is directly impacted, and it is not always possible to neglect the error introduced. For instance, assume that the generation of a dataset involves an interpolation step. Then, the interpolation error has to be taken into account during the performance evaluation of an algorithm on these synthetic data.

There is no universal procedure for evaluating the quality and performance of an interpolation method. A non-rigorous first possibility is to have a qualitative approach with a visual analysis of the results. A second standard procedure consists in interpolating samples for which an underlying continuous signal is known. However, there is no satisfactory and practical model for real-world images. A third procedure, which does not require a ground truth model, is to measure the error after applying successively several transformations whose composition is the identity (for instance a transformation and its inverse). The accuracy of the interpolation method is not directly measured, but this gives an information about the reversibility (or stability) of the interpolation method. For instance, in [13, Section VIII] 15 rotations of angle $\frac{2 \pi}{15}$ are applied. Similarly in [9], the authors perform forward and backward image transformations by optical flow.

Even though the Shannon-Whittaker formula cannot be used in practice, images are still commonly assumed to be band-limited. The interpolation error is interpreted as the consequence of the cardinal sine approximation by the interpolation kernel. The Fourier transform of the kernel is, in general, a smooth approximation of the Nyquist domain indicator function $[13,8,5]$. In practice, for finite images, the Shannon-Whittaker interpolate becomes a trigonometric polynomial when the band-limitedness assumption is made along with a periodic extension [1]. As shown in [4], a trigonometric polynomial interpolation is compatible with DFT-based computations and can be used in practice. It provides high quality results because it does not introduce spectral attenuation or aliasing in the high-frequencies. As pointed out in [1], it is possible to improve the performance of interpolation methods using a DFT zoom-in i.e. an up-sampling by trigonometric polynomial interpolation. However, the underlying periodic assumption is not compatible in general with the image content and this may introduce undesirable ringing artifacts. This led the author of [10], to apply a periodic plus smooth decomposition for handling the non-periodicity during the up-sampling of images. The periodic component is up-sampled using a DFT zoom-in while the (very) smooth component is interpolated by bilinear interpolation.

In this work, we introduce a new quantity for evaluating the interpolation method quality: the reversibility error. For a given image and a homography assumed to be close to the identity, the error after applying successively the homography, a crop (removing boundary artifacts) and the inverse homography is measured. The reversibility error is estimated by averaging the error over a large number of random homographies. A more precise measurement discarding very high-frequency artifacts is obtained by a minor clipping of the spectrum of the difference. We also propose new fine-tuned interpolation methods that are based on the DFT zoom-in and pre-existing (or base) interpolation methods. The zoomed version of an interpolation method is obtained by applying it to the DFT zoom-in of the image. In the periodic plus smooth version of interpolation methods, the non-periodicity is handled by applying the zoomed version to the periodic component and a base interpolation method to the smooth component. In an experimental part, we show that the proposed fine-tuned methods have smaller reversibility errors than their base interpolation methods and that the error is mainly localized in a small high-frequency band.

This work is based on [3, Chapter 6] and is organized as follows. First, the reversibility errors are defined in Section 2. Then, the new fine-tuned methods are introduced in Section 3. Finally, the performance of these methods are experimentally compared in Section 5. 
Remark. In the following only grayscale images are considered. The results are trivially extended to color (or multi-channel) images by handling channels independently.

\section{Reversibility Error of Interpolation Methods}

In the rest of this work, $M$ and $N$ denote two non-negative integers, $\underline{u}$ denotes a real-valued image of size $M \times N$.

Two variants of the reversibility error of an interpolation method are introduced in Section 2.3. But first, the homographies used for evaluating the reversibility errors are presented in Section 2.1. Then, spectrum clipping is introduced in Section 2.2 as it is an important correction of the reversibility error.

\subsection{Moderate Homographies}

As it is used for evaluating the reversibility error, the transformation of an image using an interpolation method is first defined.

Definition 1 (Spatial domain). The discrete spatial domain $\Omega_{M, N}$ is defined by

$$
\Omega_{M, N}=\{0, \ldots, M-1\} \times\{0, \ldots, N-1\} .
$$

Definition 2 (Geometric transformation of an image). The transformation of the image $\underline{u}$ of size $M \times N$ by a one-to-one function $\varphi: \mathbb{R}^{2} \rightarrow \mathbb{R}^{2}$ (called geometric transformation) is noted $\underline{u}_{\varphi}$ and is defined by

$$
\forall(k, l) \in \Omega_{M, N}, \quad\left(\underline{u}_{\varphi}\right)_{k, l}=u\left(\varphi^{-1}(k, l)\right) .
$$

The real number $u(x, y)$ in (2) denotes the interpolated value at location $(x, y) \in \mathbb{R}^{2}$ of $\underline{u}$ obtained with an interpolation method, which is specified in the context. Note that when $(x, y) \notin[0, M-1] \times[0, N-1]$ a boundary extension (e.g. periodic, constant, half-symmetric or whole-symmetric) is used.

As discussed later, the transformations used during the reversibility error evaluation have to be close to the identity in order to avoid the unnecessary introduction of boundary errors and of aliasing. Therefore, we define and use "moderate" homographies.

Definition 3 (Moderate homographies). For a given image $\underline{u}$ of size $M \times N$, a homography $\varphi: \mathbb{R}^{2} \rightarrow$ $\mathbb{R}^{2}$ is said to be "moderate" if the displacements of the four corners $C_{1}=(0,0), C_{2}=(M-1,0)$, $C_{3}=(0, N-1)$ and $C_{4}=(M-1, N-1)$ are smaller 1 along each dimension. In other words,

$$
\varphi \text { is a moderate homography } \Leftrightarrow \quad \text { for } i \in\{1,2,3,4\}, \quad C_{i}-\varphi\left(C_{i}\right) \in[-1,1]^{2} \text {. }
$$

Moderate homographies can be easily generated by moving the four corners of the image using Algorithm 1 with $L=1$. Note that increasing the maximal allowed displacement may introduce additional errors uniquely due to the choice of the transformation (and not the interpolation method).

\subsection{Spectrum Clipping}

In this section the spectrum clipping, an important correction of the reversibility error, is defined.

The spectrum of a discrete image is obtained thanks to its discrete Fourier transform (DFT), which is defined as follows. 


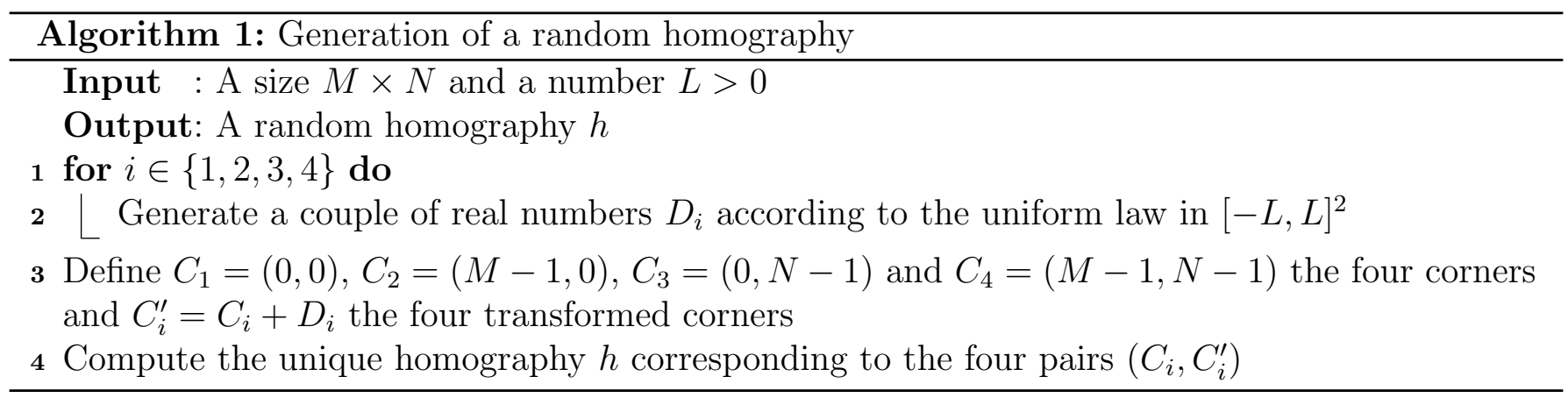

Definition 4 (Fourier domain). The discrete Fourier domain $\hat{\Omega}_{M, N}$, associated to $\Omega_{M, N}$, is defined by $\hat{\Omega}_{M, N}=\hat{\Omega}_{M} \times \hat{\Omega}_{N}$ where for a positive integer $L$

$$
\hat{\Omega}_{L}= \begin{cases}\left\{-\frac{L-1}{2}, \ldots, \frac{L-1}{2}\right\} & \text { if } L \text { is odd } \\ \left\{-\frac{L}{2}, \ldots, \frac{L}{2}-1\right\} & \text { if } L \text { is even. }\end{cases}
$$

Definition 5 (Discrete Fourier transform). The discrete Fourier transform (DFT) of $\underline{u} \in \mathbb{C}^{\Omega_{M, N}}$ is denoted $\mathcal{F}_{M, N}(\underline{u}) \in \mathbb{C}^{\hat{\Omega}_{M, N}}$ and is defined by

$$
\forall(m, n) \in \hat{\Omega}_{M, N}, \quad \mathcal{F}_{M, N}(\underline{u})_{m, n}=\sum_{(k, l) \in \Omega_{M, N}} \underline{u}_{k, l} e^{-2 \pi i\left(k \frac{m}{M}+l \frac{n}{N}\right)} .
$$

The inverse discrete Fourier transform (iDFT) of $\underline{v} \in \mathbb{C}^{\hat{\Omega}_{M, N}}$ is denoted $\mathcal{F}_{M, N}^{-1}(\underline{v}) \in \mathbb{C}^{\Omega_{M, N}}$ and is defined by

$$
\forall(k, l) \in \Omega_{M, N}, \quad \mathcal{F}_{M, N}^{-1}(\underline{v})_{k, l}=\frac{1}{M N} \sum_{(m, n) \in \hat{\Omega}_{M, N}} \underline{v}_{m, n} e^{2 \pi i\left(m \frac{k}{M}+n \frac{l}{N}\right)} .
$$

Let $r \in[0,1]$. The spectrum of the image $\underline{u}$ is clipped with ratio $r$ as follows. For $L$ a positive integer, denote by $c_{L, r}$ the piece-wise constant function defined by

$$
c_{L, r}(x)= \begin{cases}1 & |x| \leq(1-r) L / 2 \\ 0 & |x|>(1-r) L / 2\end{cases}
$$

Definition 6 (Spectrum clipping). The image $\underline{u}^{c}$, whose spectrum corresponds to the spectrum of $\underline{u}$ clipped with ratio $r \in[0,1]$, is the image of size $M \times N$ defined by

$$
\mathcal{F}_{M, N}\left(\underline{u}^{c}\right)_{m, n}=\mathcal{F}_{M, N}(\underline{u})_{m, n} c_{M, r}(m) c_{N, r}(n) .
$$

Spectrum clipping can be seen as a (perfect) lowpass filtering operation. Along each dimension only the frequencies lower or equal to $(1-r) \pi$ are kept. The DFT coefficient $\mathcal{F}_{M, N}(\underline{u})_{m, n}$ is killed if and only if

$$
c_{M, r}(m) c_{N, r}(n)=0 \quad \text { if and only if } \quad\left(\frac{2 \pi m}{M}>(1-r) \pi \text { or } \frac{2 \pi n}{N}>(1-r) \pi\right) .
$$

Note that because of the Parseval Theorem, spectrum clipping reduces the energy (i.e. the root mean square) of the images. To simplify, the dependency of $\underline{u}^{c}$ on $r$ is skipped in the notation. For $r=0$ spectrum clipping has no impact i.e. $\underline{u}^{c}=\underline{u}$. For $r=1, \underline{u}^{c}$ is the constant image corresponding to the mean of $\underline{u}$. An example of spectrum clipping with $r=1 \%$ is shown in Figure 1. 


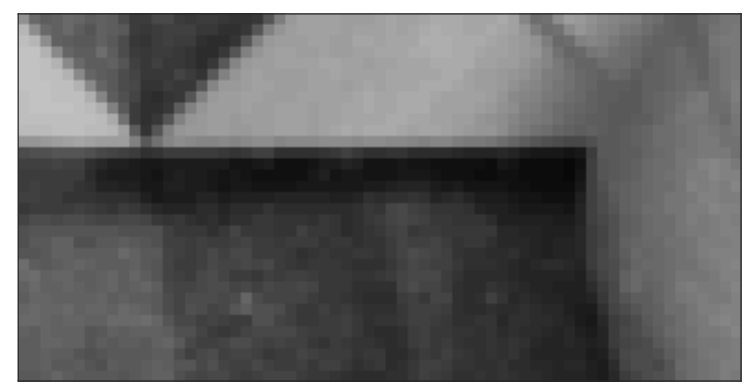

(a) Input image $\underline{u}$

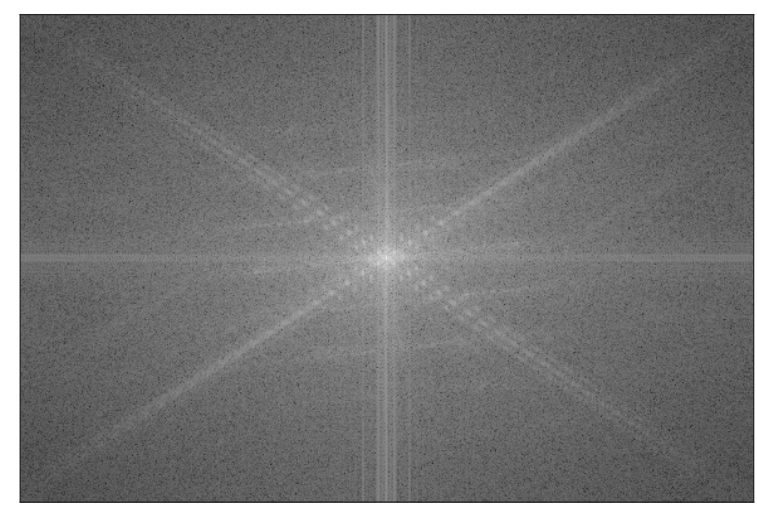

(c) Spectrum of (a)
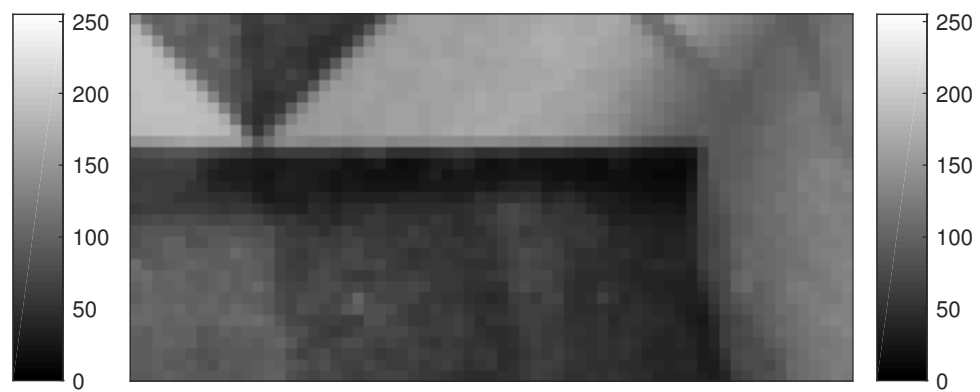

(b) $\underline{u}^{c}$
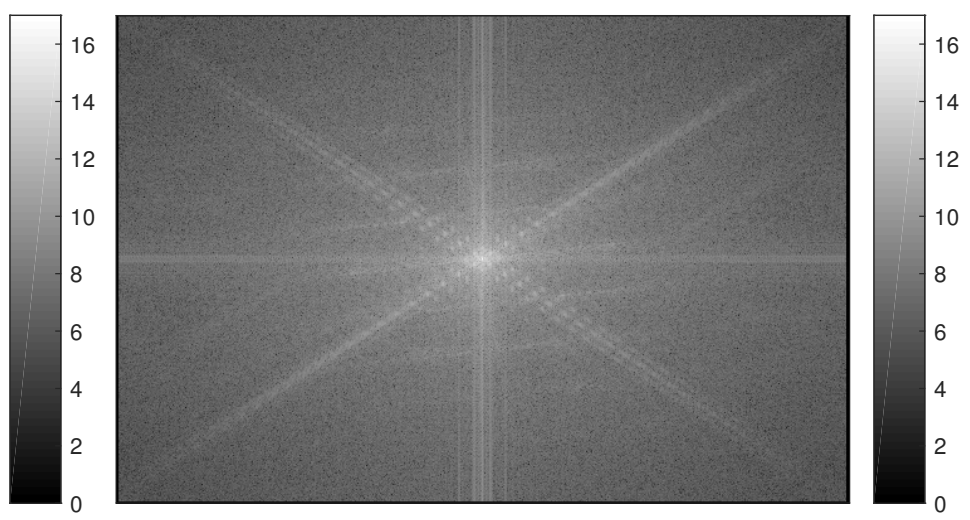

(d) Spectrum of (b)

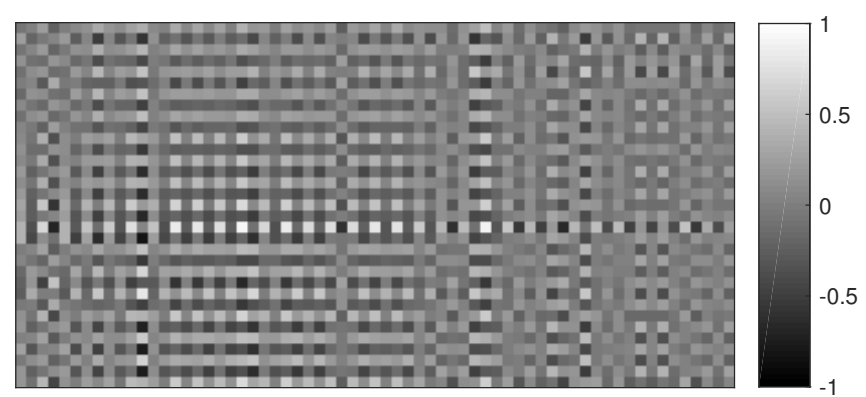

(e) Difference between (a) and (b)

Figure 1: Example of spectrum clipping using $r=1 \%$ on the RubberWhale image. Only details of the images are shown in (a), (b) and (e). The difference (e) between (a) and (b) is not visible to the naked eye. The spectra in (c) and (d) correspond to the discrete Fourier transform in logarithmic scale $x \mapsto \log (1+x)$. The RMSE between $\underline{u}$ and $\underline{u}^{c}$ is approximately 0.29 . The energy (i.e the root mean square) of $\underline{u}$ is around 143 so that the ratio of energy removed is approximately of $0.2 \%$. 


\subsection{Definition and Evaluation of the Reversiblity Errors}

In the following $\varphi$ is assumed to be a moderate homography (see Definition 3 ). Let $\delta$ be a nonnegative integer that is assumed to be small with respect to the size of the image (e.g. $\delta=20$ ). Denote by $C_{\delta}$ the crop operator of size $\delta$ so that $C_{\delta}(\underline{u})$ is the image of size $(M-2 \delta) \times(N-2 \delta)$ verifying

$$
\left(C_{\delta}(\underline{u})\right)_{k, l}=\underline{u}_{k+\delta, l+\delta} .
$$

The image $\underline{\tilde{u}}(\varphi)=\underline{\tilde{u}}(\varphi, \delta)$ is defined as the image of size $(M-2 \delta) \times(N-2 \delta)$ verifying

$$
\underline{\tilde{u}}(\varphi)=\left(C_{\delta}\left(\underline{u}_{\varphi}\right)\right)_{\varphi^{-1}} .
$$

The image $\underline{\tilde{u}}(\varphi)$ represents the reconstruction of the image $\underline{u}$ after successively applying the moderate homography $\varphi$ and its inverse $\varphi^{-1}$. For $\delta=0, \underline{\tilde{u}}(\varphi)=\underline{\tilde{u}}(\varphi, 0)=\left(\underline{u}_{\varphi}\right)_{\varphi^{-1}}$. For $\delta>0$, the homography $\varphi^{-1}$ is applied to the cropped transformed image $C_{\delta}\left(\underline{u}_{\varphi}\right)$. The aim of the crop is to get rid of boundary artifacts introduced during the first transformation.

The difference image $D I(\varphi)=D I(\varphi, \underline{u}, \delta)$ is defined as the image of size $(M-4 \delta) \times(N-4 \delta)$ verifying

$$
D I(\varphi)=C_{\delta}(\underline{\tilde{u}}(\varphi))-C_{2 \delta}(\underline{u}) .
$$

$D I(\varphi)$ is nothing but the cropped difference image between the reconstructed image $\underline{\tilde{u}}(\varphi)$ and the input image $\underline{u}$. Here, the aim of the crop is to prevent from considering the boundary artifacts introduced during the second transformation.

Note that as soon as $\varphi$ is not the identity the computation of $\underline{\tilde{u}}(\varphi)$ requires the use of a boundary extension (for at least one of the two transformations). The crop is sufficient to discard the influence of the boundary extension.

Definition 7 (Reversibility error for a homography). Assume that $\varphi$ is a moderate homography. Then, the reversibility error for the homography $\varphi$, noted $\mathcal{E}(\varphi)$, is defined as the $\ell^{2}$ energy of the difference image $D I(\varphi)$ (see (12)). It is given by

$$
\mathcal{E}(\varphi)^{2}=\frac{1}{(M-4 \delta)(N-4 \delta)} \sum_{k=0}^{(M-1-4 \delta)} \sum_{l=0}^{(N-1-4 \delta)}\left|D I(\varphi)_{k, l}\right|^{2} .
$$

For $\delta=0$, the reversibility error $\mathcal{E}(\varphi)$ corresponds to the RMSE between the reconstructed image $\underline{\tilde{u}}(\varphi)$ and $\underline{u}$. Note that to simplify the dependencies on the image $\underline{u}$, the interpolation method and $\delta$ are not specified.

Clipped reversibility error for a homography. A variant is obtained by replacing $D I(\varphi)$ by its clipped version $D I(\varphi)^{c}$. The spectrum clipping of $D I(\varphi)$ is done with ratio $r=1 \%$ as described in Section 2.2. This variant is called the clipped reversibility error for the homography $\varphi$ and is denoted by $\mathcal{E}^{c}(\varphi)$. Both measurements are linked by the inequality $\mathcal{E}^{c}(\varphi) \leq \mathcal{E}(\varphi)$ by Parseval's theorem.

Note that depending on the geometric transformation, applying it to an image may introduce aliasing. For transformations close to the identity, such as moderate homographies, the aliasing is localized in a small high-frequency band. The clipped reversibility error is built in order to discard the influence of this aliasing on the interpolation quality evaluation.

Reversibility errors. The quantities $\mathcal{E}(\varphi)$ and $\mathcal{E}^{c}(\varphi)$ depend on the choice of $\varphi$. Assume that $\varphi$ is a random moderate homography generated by moving the four corners of the image using Algorithm 1 with $L=1$. Then, $\mathcal{E}(\varphi)$ and $\mathcal{E}^{c}(\varphi)$ are random variables whose expected values do not depend on a particular homography. 
Definition 8 (Reversibility errors). Let $\varphi$ be a random moderate homography generated using Algorithm 1 with $L=1$. The reversibility error, noted $\mathcal{E}$, is defined as the expected value of $\mathcal{E}(\varphi)$. Similarly, the clipped reversibility error, noted $\mathcal{E}^{c}$, is defined as the expected value of $\mathcal{E}^{c}(\varphi)$.

Estimation of the reversibility errors. The estimation of the reversibility errors is summarized in Algorithm 2. The expected values $\mathcal{E}$ and $\mathcal{E}^{c}$ are estimated by computing the average of the reversibility errors over a large number $N_{\text {transf }}$ (e.g. $\left.N_{\text {transf }}=1000\right)$ of random moderate homographies. We have

$$
\mathcal{E} \simeq \frac{1}{N_{\text {transf }}} \sum_{i=1}^{N_{\text {transf }}} \mathcal{E}\left(\varphi_{i}\right)
$$

and

$$
\mathcal{E}^{c} \simeq \frac{1}{N_{\text {transf }}} \sum_{i=1}^{N_{\text {transf }}} \mathcal{E}^{c}\left(\varphi_{i}\right)
$$

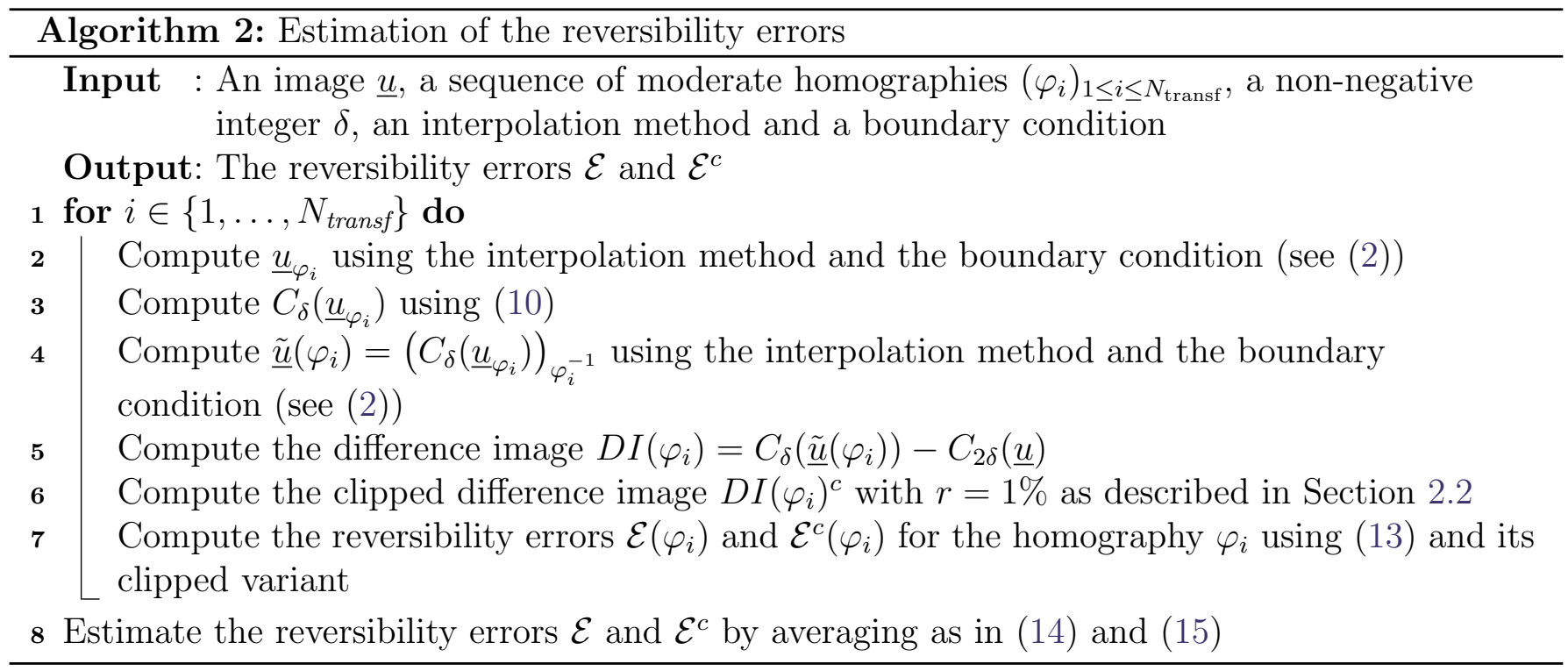

Interpretation. The difference measured by the reversibility errors is partly the consequence of a loss of information that depends on the image content and the transformation. A transformed image has the same size as the input image so that, in the spatial domain, information may be lost at the boundaries during the first transformation. Missing outside samples are replaced by arbitrary values during the second transformation, which causes what is called truncation error [12]. The sampling rate is unchanged when an image is transformed so that, in the Fourier domain, the spectrum may go outside of the Nyquist domain $[-\pi, \pi]^{2}$. Thus, a well-sampled image may become under-sampled. For instance applying a scaling by a factor greater than 1, i.e. a dilation, results in an over-sampled image but some regions of the original images are lost. On the contrary applying a scaling by a factor smaller than 1, i.e. a contraction, results in an under-sampled image with additional regions whose contents depend on the boundary extension.

By restricting the study to moderate homographies it is possible to limit the loss of information (coming from the transformation) and to analyze the impact of the interpolation method choice on the difference. As shown in Section 5, the difference caused by the interpolation method is mainly localized in the high-frequencies. Indeed, the interpolation kernel is in general an approximation 
of the cardinal sine. It does not reconstruct properly the high-frequencies and may introduce an unwanted blur.

The reversibility errors $\mathcal{E}$ and $\mathcal{E}^{c}$ can be seen as lower bounds for many performance evaluation processes using a ground truth structure. They provide a quantitative evaluation of the interpolation method stability. In particular, the difference between a reference image and a registered image of the same scene (in terms of intensity) is due to the registration error and the interpolation error (assuming there is no noise and no lighting change). In the ideal case of perfect registration and synthetic data, the error is directly linked to the reversibility error of the interpolation method for this image.

\section{Fine-tuned Interpolation Methods}

In this section fine-tuned interpolation methods are built from pre-existing methods, which are called "base" methods. They rely on the DFT zoom-in i.e. the up-sampling by trigonometric polynomial interpolation. In the following we use the trigonometric polynomial interpolator in real convention. See [4] for more details.

\subsection{Zoomed Version of Interpolation Methods}

As pointed out in [1], it is possible to improve the performance of interpolation methods using a DFT zoom-in. Consider a base interpolation method and let us describe the corresponding zoomed version. Let $\lambda>1$ be a rational number representing a zoom factor and denote by $\underline{u}^{\lambda}$ the DFT zoom-in of $\underline{u}$ by factor $\lambda[4]$. The interpolate of $\underline{u}$ using the zoomed method is defined as the rescaling by factor $\frac{1}{\lambda}$ of the interpolate of $\underline{u}^{\lambda}$ using the base method. In other words, for $(x, y) \in \mathbb{R}^{2}$, the interpolated value $u(x, y)$ is given by

$$
u(x, y)=u^{\lambda}(\lambda(x, y))
$$

where $\underline{u}^{\lambda}$ is interpolated at location $\lambda(x, y)$ using the base interpolation method. The geometric transformation of an image using a zoomed interpolation method is described in Algorithm 3.

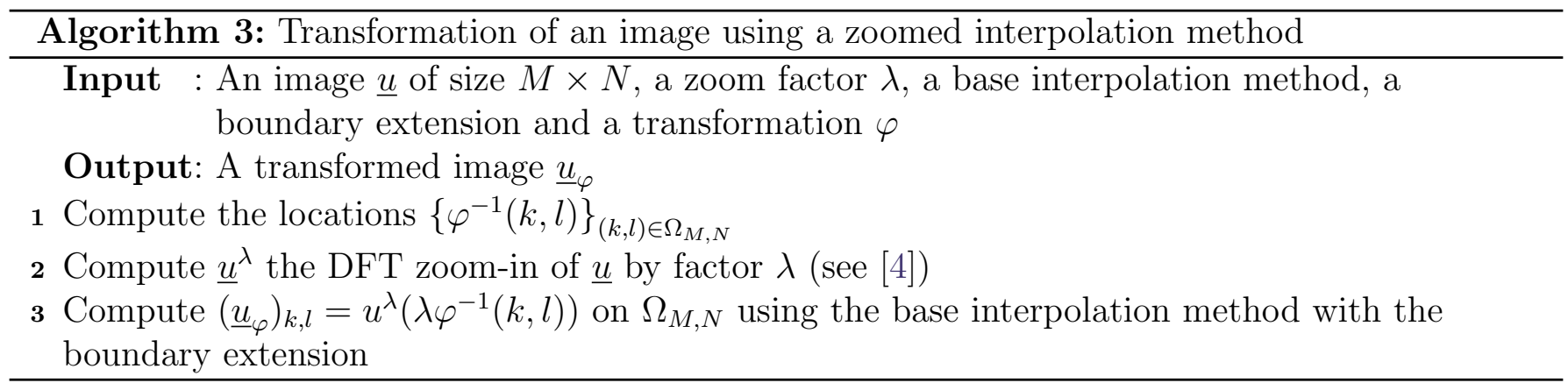

The zoomed version is computationally less efficient than its base interpolation method since it requires to zoom in the image and to compute the representation of the zoomed in image for the base interpolation method. As shown in Section 5, the zoomed versions have smaller reversibility errors than their base methods. Intuitively, they are closer to the discrete Shannon interpolation method and the base interpolation method is applied to a well-sampled image. However the DFT zoom-in relies on trigonometric polynomial interpolation and assumes a periodic extension, which may not be adapted to the image content and may introduce ringing artifacts. 


\subsection{Periodic plus Smooth Decomposition Version of Interpolation Meth- ods}

Introduced in [10], the periodic plus smooth decomposition is useful for handling the non-periodicity of images $[1,6,7]$. The image $\underline{u}$ is decomposed into a periodic component $p$, which is almost periodic, and a smooth component $\underline{s}$, which varies slowly. The images $\underline{p}$ and $\underline{s}$ (of size $M \times N$ ) are linked by the relation $\underline{u}=\underline{p}+\underline{s}$ and $\underline{p}$ has the same mean as $\underline{u}$.

Periodic plus smooth decomposition of an image. More precisely, the decomposition is characterized and computed as follows. Denote by $\underline{v}_{1}$ and $\underline{v}_{2}$ the two images of size $M \times N$ defined by

$$
\left(\underline{v}_{1}\right)_{k, l}= \begin{cases}\underline{u}(M-1-k, l)-\underline{u}(k, l) & \text { if } k=0 \text { or } k=M-1, \\ 0 & \text { otherwise }\end{cases}
$$

and

$$
\left(\underline{v}_{2}\right)_{k, l}= \begin{cases}\underline{u}(k, N-1-l)-\underline{u}(k, l) & \text { if } l=0 \text { or } l=N-1, \\ 0 & \text { otherwise. }\end{cases}
$$

Set $\underline{v}=\underline{v}_{1}+\underline{v}_{2}$. Denote by $\Delta$ the discrete Laplacian operator that associates to an image $\underline{w}$ of size $M \times N$ the image $\Delta \underline{w}$, of the same size, defined by

$$
\Delta \underline{w}=K_{\text {lap }} \star \underline{w}
$$

where $\star$ represents the $(M, N)$-periodic convolution operator and

$$
K_{\text {lap }}=\left[\begin{array}{ccc}
0 & 1 & 0 \\
1 & -4 & 1 \\
0 & 1 & 0
\end{array}\right]
$$

Then, the periodic component $\underline{p}$ of $\underline{u}$ is defined as the unique solution of the discrete Poisson equation

$$
\left\{\begin{array}{l}
\Delta \underline{p}=\Delta \underline{u}-\underline{v} \\
\operatorname{mean}(\underline{p})=\operatorname{mean}(\underline{u}) .
\end{array}\right.
$$

In practice the decomposition is obtained using DFT-based computations. Indeed, the DFT of the smooth component $\underline{s}$ is given by $\mathcal{F}_{M, N}(\underline{s})_{0,0}=0$ (zero mean) and

$$
\forall(m, n) \in \hat{\Omega}_{M, N} \backslash(0,0), \quad \mathcal{F}_{M, N}(\underline{s})_{m, n}=\frac{\mathcal{F}_{M, N}(\underline{v})_{m, n}}{2 \cos \left(\frac{2 \pi m}{M}\right)+2 \cos \left(\frac{2 \pi n}{N}\right)-4} .
$$

An example of decomposition is shown in Figure 2. The cross structure in the spectrum of $\underline{u}$, which is caused by its non-periodicity, is not visible in the spectrum of $\underline{p}$.

Periodic plus smooth version of interpolation methods. In [10], this periodic plus smooth decomposition is used to up-sample images. More generally, the decomposition can be used to improve interpolation methods as follows.

The periodic component $p$ is almost periodic so that the periodic extension of its DFT zoom-in does not introduce undesirable ringing artifacts. It is interpolated using a zoomed version of an interpolation method with periodic extension. The smooth component $\underline{s}$ varies slowly and is easily interpolated by a base interpolation method with any extension. Each component is interpolated independently, possibly using different base interpolation methods, and the results are added to get the interpolated values. 


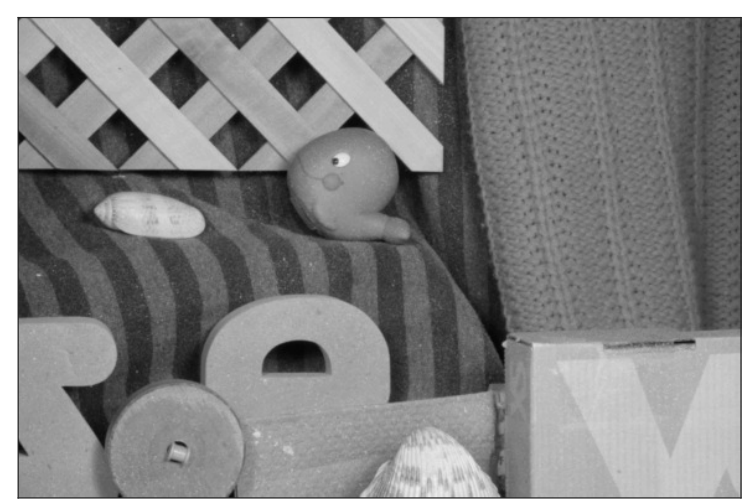

(a) Input image $\underline{u}$

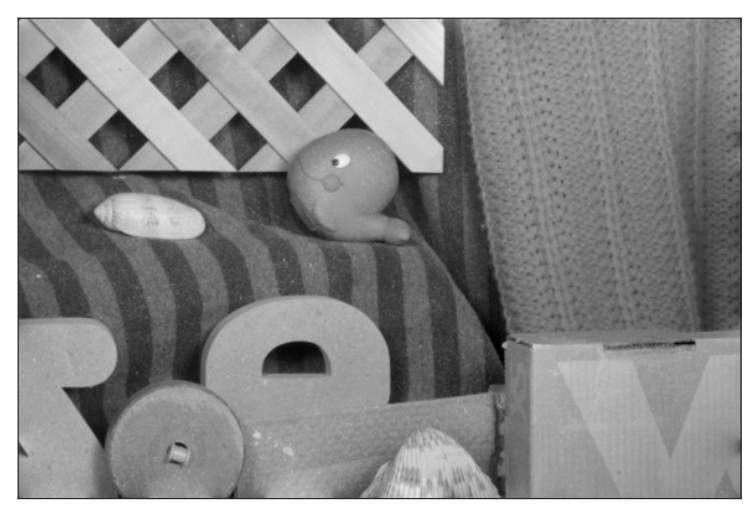

(c) Periodic component $\underline{p}$

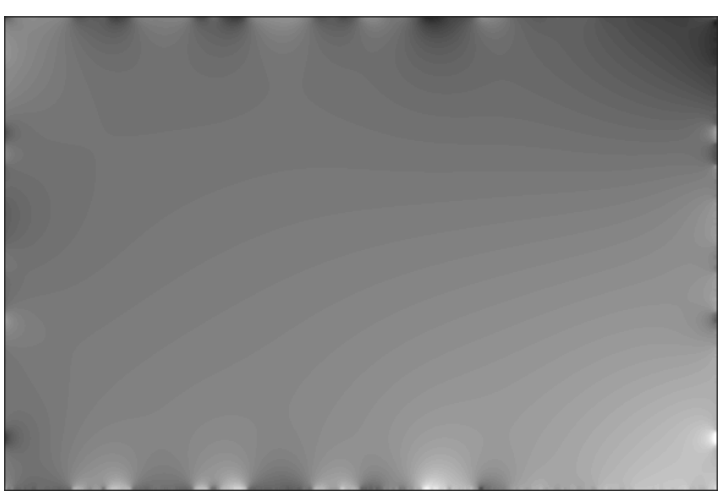

(e) Smooth component $\underline{s}$
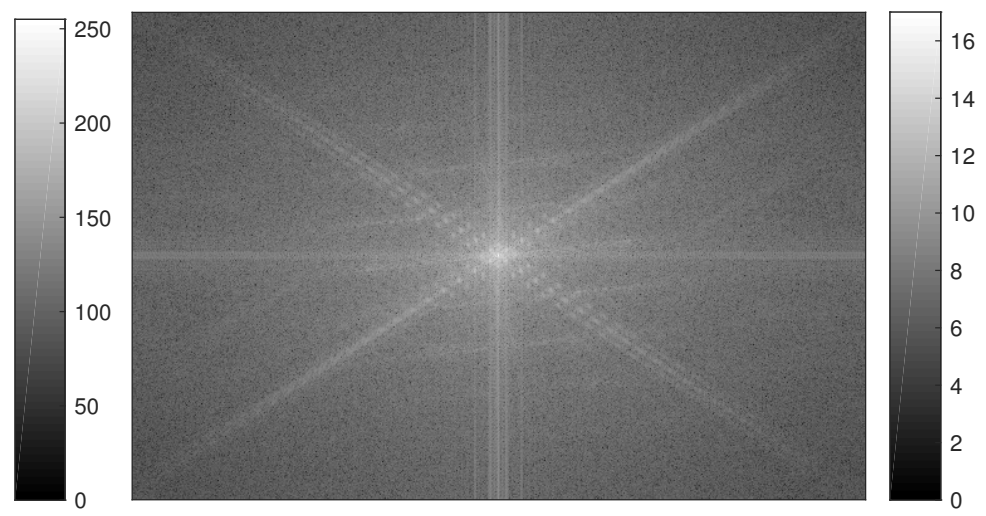

(b) Spectrum of $\underline{u}$
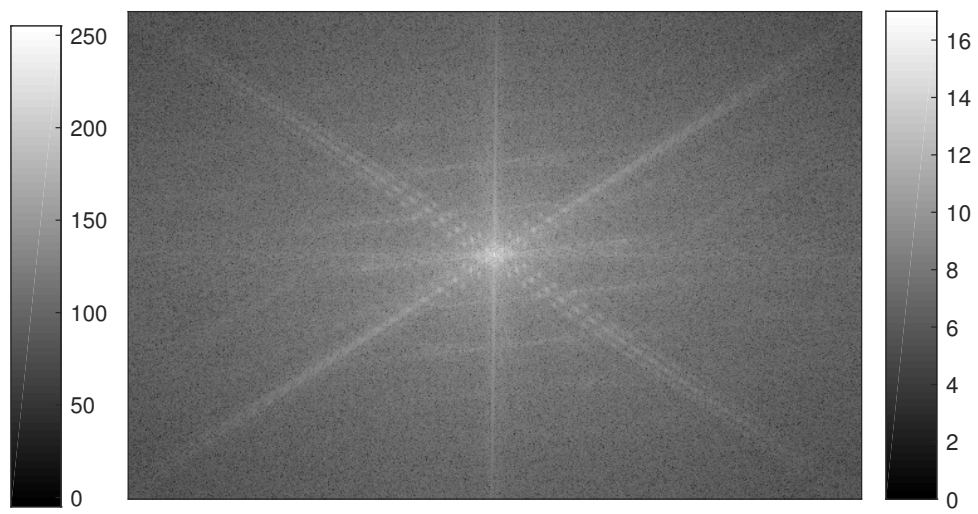

(d) Spectrum of $\underline{p}$

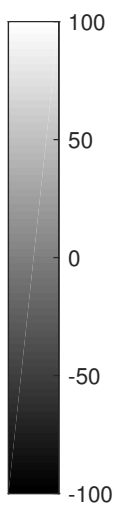

$-100$

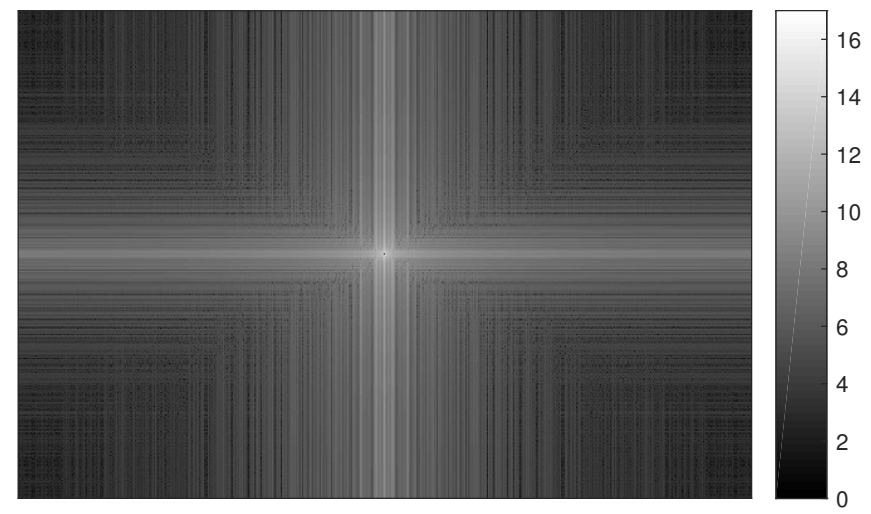

(f) Spectrum of $\underline{s}$

Figure 2: Periodic plus smooth decomposition $\underline{u}=\underline{p}+\underline{s}$ of the RubberWhale image. The spectra in (b), (d) and (f) correspond to the discrete Fourier transform in logarithmic scale $x \mapsto \log (1+x)$. The content of the periodic component $p$ is almost periodic and is similar to the input except near the image boundaries. The smooth component $\underline{s}$ varies slowly and is close to 0 in the center of the image. The cross structure in the spectrum of $\underline{u}$, which is caused by the non-periodicity, is not visible in the spectrum of $\underline{p}$. 
Algorithm. The transformation of an image using the periodic plus smooth version of interpolation methods is described in Algorithm 4. As the periodic plus smooth decomposition is computed using DFT-based computations (see (22)), in practice the DFT zoom-in of $\underline{p}$ is obtained directly from $\mathcal{F}_{M, N}(\underline{p})$, which saves the computations of a DFT and an iDFT.

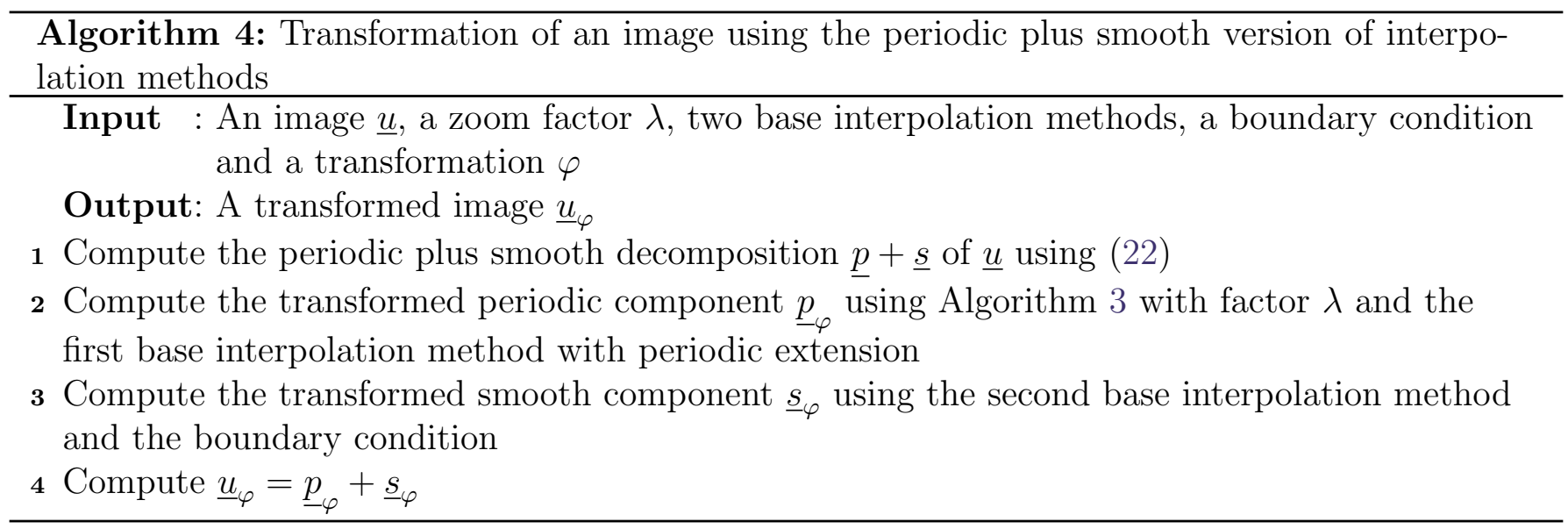

\section{Provided Implementation and Online Demo}

The source codes and an online demo are available at the IPOL web page of this article ${ }^{2}$. We propose ANSI C99 implementations of the interpolation methods considered in Section 5. In particular, we propose implementations of the zoomed versions and periodic plus smooth versions as described in Algorithm 3 and Algorithm 4.

In the online demo the user is invited to upload an image $\underline{u}$ and to choose a crop size $\delta$, a spectrum clipping ratio $r$, a homography $\varphi$ and an interpolation method (with a given boundary condition). Then, the demo computes the transformed image $\underline{u}_{\varphi}$, the difference image $D I(\varphi)$ and its spectrum, and the corresponding reversibility errors $\mathcal{E}(\varphi)$ and $\mathcal{E}(\varphi)^{c}$.

\section{$5 \quad$ Experiments}

In this experimental part, interpolations methods are compared in terms of reversibility errors and efficiency. The interpolation methods used are the following:

- Five base interpolation methods: trigonometric polynomial interpolation, bicubic interpolation and B-spline interpolations of order 1, 3 and 11. They are noted respectively tpi, bic, spline1, spline3 and spline11. Note that spline1 corresponds to the so-called bilinear interpolation. For the trigonometric polynomial interpolation we use the efficient algorithm of [4] in real convention. It is an approximate (but precise to the machine precision) algorithm based on the nonnequispaced fast Fourier transform (NFFT) [11].

- The zoomed versions, with zoom factor $\lambda=2$, of the base interpolation methods (except for tpi because by construction the DFT zoom-in is useless). The suffix $z 2$ is added to the corresponding base method.

\footnotetext{
${ }^{2}$ https://doi.org/10.5201/ipol.2019.277
} 
- The periodic plus smooth versions, with zoom factor $\lambda=2$, of several pairs of interpolation methods. The notation of the method starts with $p+s$, then the second term refers to the interpolation method used for the periodic component and an eventual third term corresponds to the interpolation method used for the smooth component (if different from the method used for the periodic component). Note that the DFT zoom-in is not applied for the $p+s-t p i_{-}^{* * *}$ methods.

For example the periodic plus smooth version of the B-spline interpolation of order 11 and the bicubic interpolation is noted $p+s$-spline11-bic. The half-symmetric extension is used for all the methods except the tpi for which the use of the periodic extension is required. The performance evaluation was made using the crop size $\delta=20$, which is negligible with respect to the size of the input image but is large enough to discard the boundary extension influence.

The conclusions on the order of the methods are clear-cut and do not depend on the choice of the image. However, note that the reversibility error increases with the amount of high-frequency content (see Appendix A). The image $\underline{u}$ considered is the grayscale version of the RubberWhale image, which is a generic color image of size $584 \times 388$ taken from the Middlebury database [2]. Only details on a small part of the images are shown. The computations were made using an $\operatorname{Intel}(\mathrm{R}) \mathrm{Xeon}(\mathrm{R}) \mathrm{CPU}$ E5-2650 @ 2.60GHz using a single thread. Multi-threading, in particular for computing FFTs, can speed the computations for the zoomed and periodic plus smooth versions.

\subsection{Estimation of the Reversibility Errors}

In this section, the reversibility errors are estimated for the different interpolation methods using Algorithm 2 with $N_{\text {transf }}=1000$ moderate random homographies. The moderate homographies were generated by moving the four corners of the image using Algorithm 1 with $L=1$.

The results are presented in Table 1. It contains the two reversibility errors for the considered interpolations methods and the computation time, in seconds, for all the transformations. Therefore the displayed time can also be interpreted as the average time, in milliseconds, required to apply a transformation and its inverse.

Base interpolation methods. Except for the tpi, the reversibility errors for the base interpolation methods are the worst and spectrum clipping did not reduce significantly the value. The measurements for the tpi are better and the errors are mainly localized in the high-frequencies since the clipped reversibility error is way lower. The resulting ranking is tpi, spline11, spline3, bic, spline1. In particular increasing the B-spline order leads to better results as shown in [5].

Zoomed versions. The zoomed versions have better reversibility errors than their corresponding base methods but are slower (approximately twice slower). As the clipped reversibility error is significantly lower (except for the spline1-z2), we deduce that the error is mainly localized in the high-frequencies. The zoomed methods are closer to the tpi. In particular, using spline11-z2 provides almost the same results as the tpi but is five times faster.

Periodic plus smooth versions. The periodic plus smooth versions have the best reversibility errors. This is due to the proper handling of the non-periodicity during the DFT zoom-in. The base method used for interpolating the zoomed periodic component has to be of high order (here tpi or spline11). The method used for the smooth component can be any base method (except the tpi) since it varies smoothly and is easily interpolable. No distinction can be made in the results. Indeed, the best results in terms of reversibility error are obtained using $p+s$-spline $11_{-* * *}$ or $p+s$-tpi-*** 


\begin{tabular}{l|l|l|l} 
& $\mathcal{E}$ & $\mathcal{E}^{c}$ & Time (s) \\
\hline spline1 & 2.35537 & 2.34856 & 138 \\
bic & 1.10570 & 1.09574 & 160 \\
spline3 & 0.70991 & 0.69487 & 171 \\
spline11 & 0.33367 & 0.30318 & 504 \\
tpi & 0.16039 & 0.09102 & 2685 \\
\hline spline1-z2 & 0.79736 & 0.78378 & 356 \\
bic-z2 & 0.21280 & 0.16668 & 372 \\
spline3-z2 & 0.16157 & 0.09427 & 403 \\
spline11-z2 & 0.16039 & 0.09102 & 807 \\
\hline$p+s-s p l i n e 1$ & 0.78967 & 0.78045 & 465 \\
$p+s-b i c$ & 0.16910 & 0.14450 & 554 \\
$p+s$-spline3-spline1 & 0.08816 & 0.04009 & 529 \\
$p+s$-spline3 & 0.08816 & 0.04009 & 548 \\
$p+s$-spline11-spline1 & $\mathbf{0 . 0 8 2 1 2}$ & $\mathbf{0 . 0 2 8 3 3}$ & $\mathbf{9 4 0}$ \\
$p+s$-spline11-spline3 & 0.08212 & 0.02833 & 982 \\
$p+s-t p i-s p l i n e 1$ & 0.08212 & 0.02833 & 8241 \\
$p+s-t p i-s p l i n e 3$ & 0.08212 & 0.02833 & 8325
\end{tabular}

Table 1: Estimation of the reversibility errors for the RubberWhale image and $N_{\text {transf }}=1000$ random moderate homographies. A crop of size $\delta=20$ was used so that the results do not depend on the boundary extension choice. The displayed time corresponds to the computation time, in seconds, for all the transformations. This can also be interpreted as the average time, in milliseconds, required to apply a transformation and its inverse. The best results in terms of reversibility error are obtained using $p+s$-spline11-*** or $p+s-t p i-* * *$ methods. Among these methods the $p+s-s p l i n e 11-s p l i n e 1$ method is the most efficient and should be preferred.

methods. Among these methods the $p+s$-spline11-spline1 method is the most efficient and should be preferred.

Clipped reversibility error. As already remarked above, for the fined-tuned methods the clipped reversibility error $\mathcal{E}^{c}$ is significantly lower than the non-clipped reversibility error $\mathcal{E}$. The amount of energy removed from $\underline{u}$ only represents $0.2 \%$ of its total energy. This means that the error is mainly localized in the high-frequencies for the fined-tuned methods but not for the base methods (except the tpi).

\subsection{Transformation by a Moderate Homography}

In this section, the analysis of Section 5.1 will be confirmed visually by considering the transformation by an arbitrary moderate homography. We define the homography $\varphi$ by moving respectively the topleft corner, the top-right corner, the bottom-left and the bottom-right corner by $(1,1),(-1,-1),(0,0)$ and $(1,1)$. It is represented by the matrix

$$
H_{\varphi}=\left(\begin{array}{ccc}
0.988884 & -0.00258398 & 1 \\
-0.00341732 & 0.992305 & 1 \\
-1.32057 .10^{-5} & -1.32057 .10^{-5} & 1
\end{array}\right)
$$

The transformed images $\underline{u}_{\varphi}$ and $\underline{\tilde{u}}(\varphi)$ were computed using an interpolation method with halfsymmetric extension. The methods considered were bic, spline11, tpi, bic-z2, spline11-z2, p+s-bic, $p+s$-spline11-spline 1 and $p+s$-tpi-spline1. 
Difference images. The difference images $D I(\varphi)$ are shown in Figure 3. For the base methods bic and spline11, we recognize the structure of the image. It is also the case for the bic-z2 and $p+s$-bic methods i.e. the fine-tuned methods using bicubic interpolation on the zoomed image. For the tpi, spline11-z2, $p+s$-spline11-spline1 and $p+s$-tpi-spline1 methods the difference is mainly composed of typical high-frequency structures.

Spectra of the difference images. The spectra of the difference images are shown in Figure 4 . For the bic, bic-z2 and $p+s$-bic methods i.e. the methods where the possibly zoomed images are interpolated by bicubic interpolation, the spectrum of the difference is high everywhere except in a small low-frequency region. For the spline11 method the corresponding region is larger. This explains why the structure of the image is visible in the difference image for these methods in Figure 3. For the tpi and spline11-z2 methods, the spectrum has non-negligible values in a band around the Nyquist frequency and in a vertical and horizontal cross structure. The cross structure is also visible in the bic-z2 method and is due to the incorrect handling of the non-periodicity. Indeed it disappears in the $p+s$-spline11-spline 1 and $p+s$-tpi-spline 1 methods.

Difference images with spectrum clipping. The difference images $D I(\varphi)^{c}$ with spectrum clipping of ratio $r=1 \%$ are shown in Figure 5. Only the results for the tpi, spline11-z2, $p+s$-spline11spline 1 and $p+s$-tpi-spline 1 methods are presented because in the other cases the clipping has no significant impact on the difference images. The error is clearly lower when the periodic plus smooth decomposition is used.

\subsection{Propagation of the Error}

In this section, we evaluate the propagation of the error after successive direct and inverse transformations. Let $\left(\varphi_{i}\right)_{1 \leq i \leq N_{\text {transf }}}$ be random moderate homographies. Define the images $\underline{u}_{i}$ for $0 \leq i \leq N_{\text {transf }}$ by

$$
\left\{\begin{array}{l}
\underline{u}_{0}=\underline{u} \\
\underline{u}_{i+1}=\left(\left(\underline{u}_{i}\right)_{\varphi_{i+1}}\right)_{\varphi_{i+1}^{-1}} \quad 0 \leq i \leq N_{\text {transf }}-1 .
\end{array}\right.
$$

The RMSE, taken at a distance $\delta=20$ of the boundary, between the $\underline{u}_{i}$ and $\underline{u}$ represents the error after $i$ successive direct and inverse transformations. The fundamental difference with the reversibility error is that no crop is done between the transformations. The clipped variant of this error is obtained by clipping the spectrum with $r=1 \%$.

The interpolation methods considered are the same as in Section 5.2 (with half-symmetric extension). The evolutions of the errors using up to $N_{\text {transf }}=50$ random homographies are shown in Figure 6. Globally the errors tend to increase with the number of transformations. To prevent an important increase of the error $p+s$-spline $11^{* * *}$ or $p+s$-tpi-*** methods must be used. The curves of $p+s$-tpi-spline 1 and $p+s$-spline11-spline 1 are indistinguishable since the results are almost the same. Otherwise a large part of the spectrum is not well reconstructed: either because the interpolation method is not able to reconstruct the high-frequencies or either because the non-periodicity is not well handled (this is apparent by the presence of a cross structure in the difference spectrum). The clipped versions of the error for the tpi, spline11-z2, $p+s$-spline11-spline1 and $p+s$-tpi-spline 1 methods are significantly smaller than the original error. We deduce that the error is mainly localized in a small high-frequency band. 


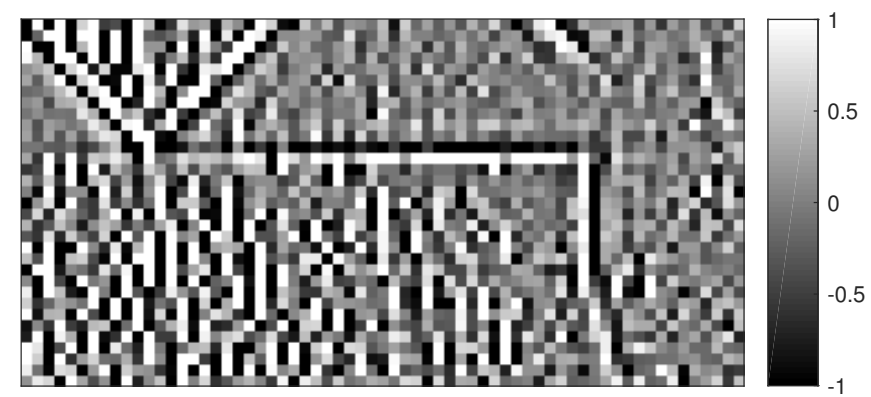

(a) bic $(0.932,0.923)$

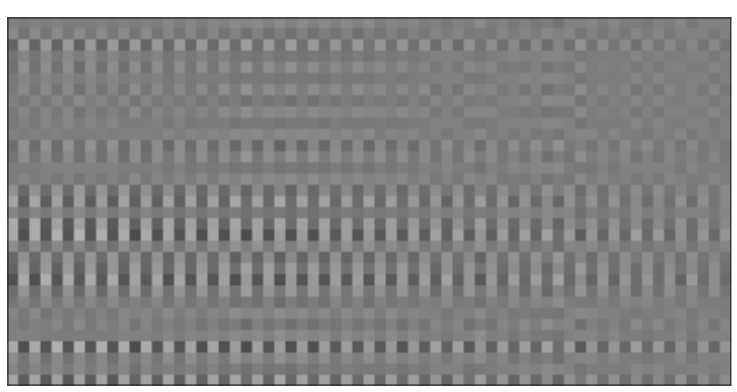

(c) tpi $(0.193,0.0877)$

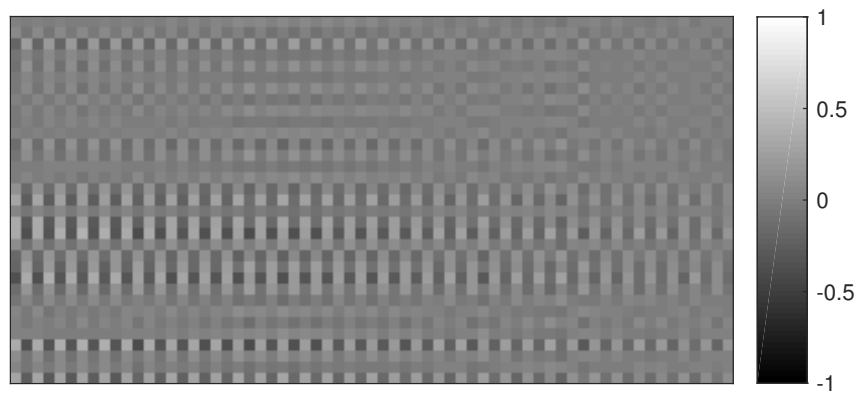

(e) spline11-z2 $(0.193,0.0877)$

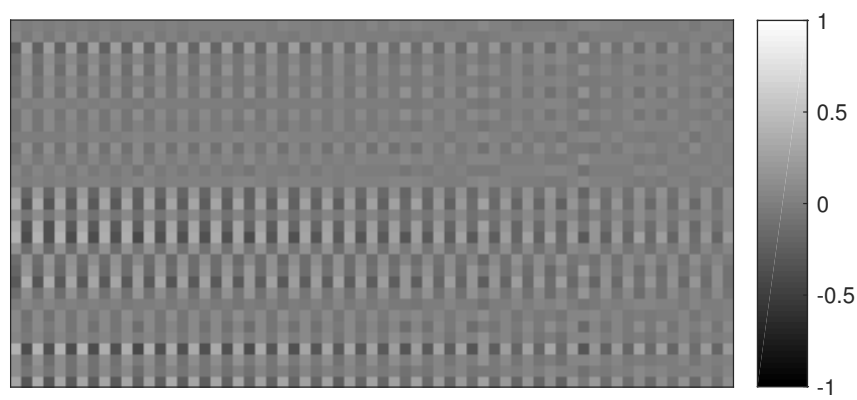

(g) $p+s$-spline11-spline1 (0.0931, 0.0372)

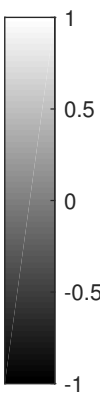

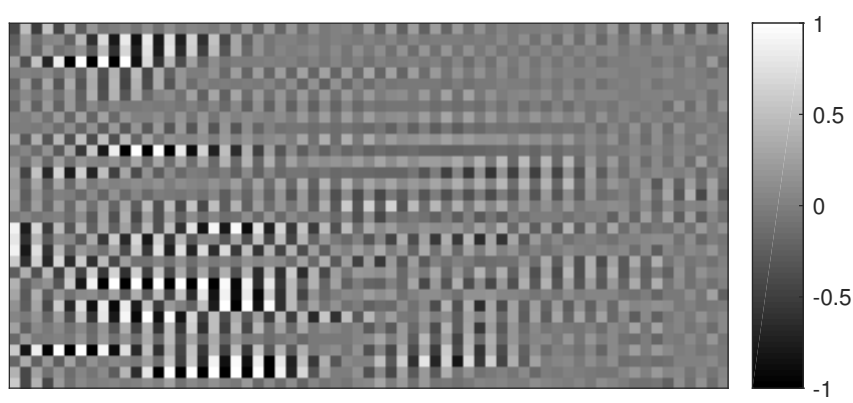

(b) spline11 $(0.280,0.254)$
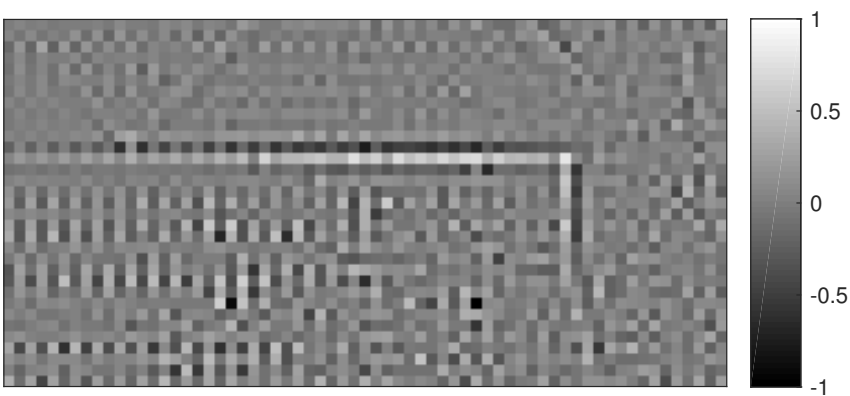

(d) bic-z2 $(0.234,0.165)$

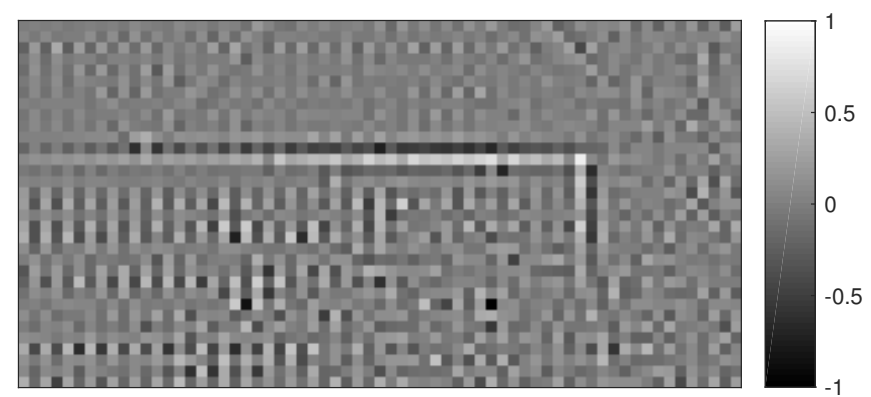

(f) $p+s$-bic $(0.172,0.146)$

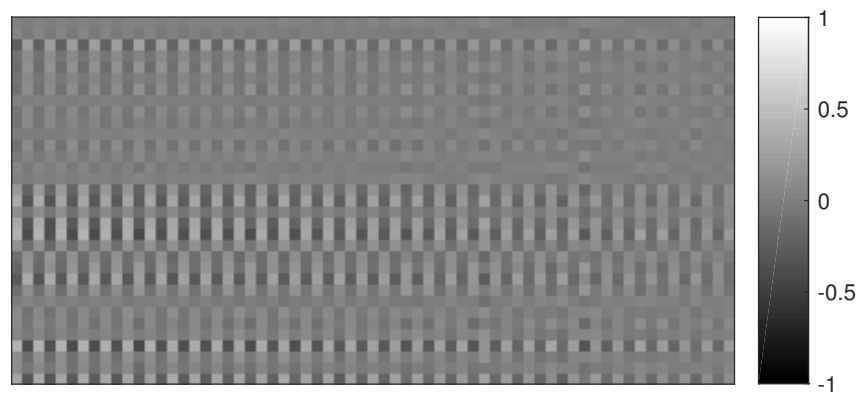

(h) $p+s$-tpi-spline1 (0.0931, 0.0372)

Figure 3: Difference image $D I(\varphi)$ for various interpolation methods. The moderate homography $\varphi$ used is defined by the matrix $H_{\varphi}$ in $(23)$. The values between parentheses correspond to $\left(\mathcal{E}(\varphi), \mathcal{E}^{c}(\varphi)\right)$. For bic and spline11, we recognize the structure of the image. It is also the case for the bic-z2 and $p+s$-bic methods i.e. the fine-tuned methods using bicubic interpolation on the zoomed image. For the tpi, spline11-z2, $p+s-s p l i n e 11-s p l i n e 1$ and $p+s-t p i-s p l i n e 1$ methods the difference is mainly composed of high-frequency structures. 


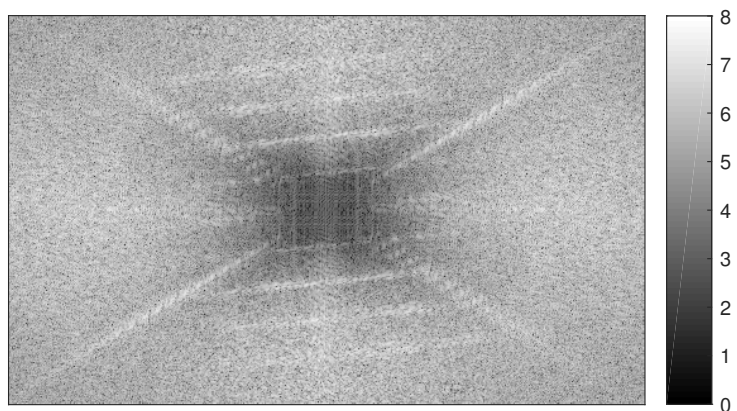

(a) bic $(0.932,0.923)$

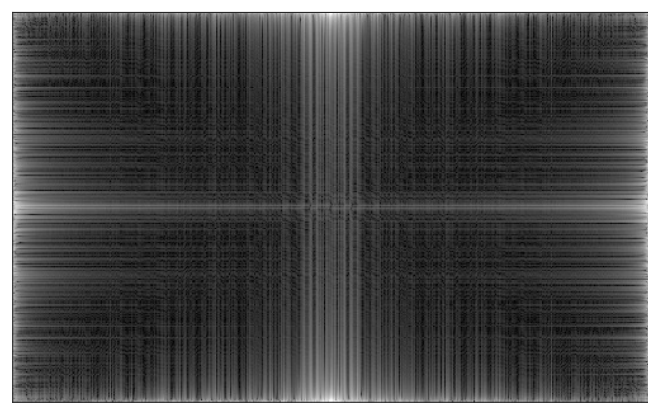

(c) $\operatorname{tpi}(0.193,0.0877)$

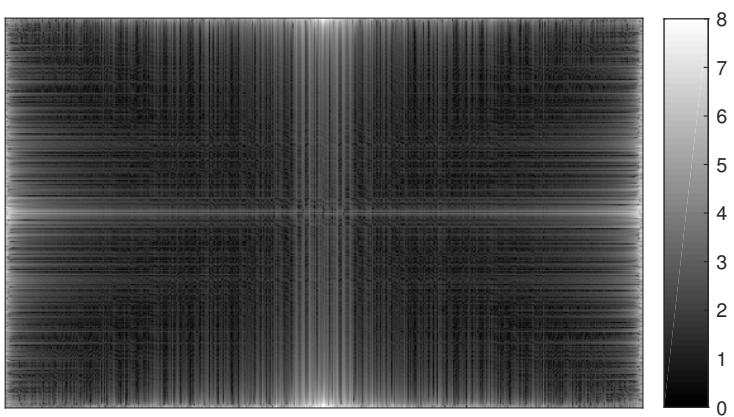

(e) spline11-z2 $(0.193,0.0877)$

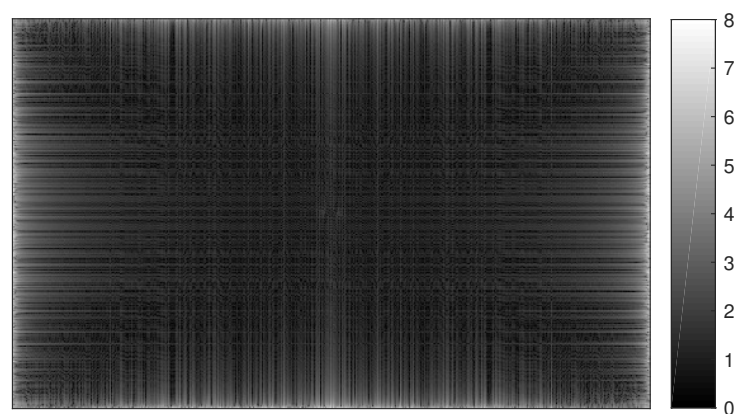

(g) $p+s$-spline11-spline1 $(0.0931,0.0372)$

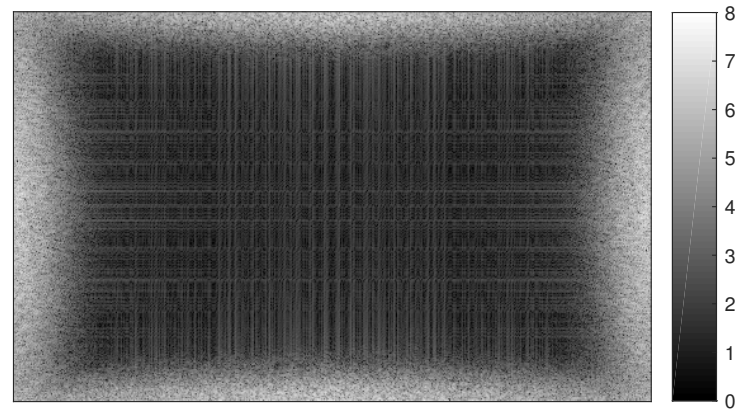

(b) spline11 $(0.280,0.254)$
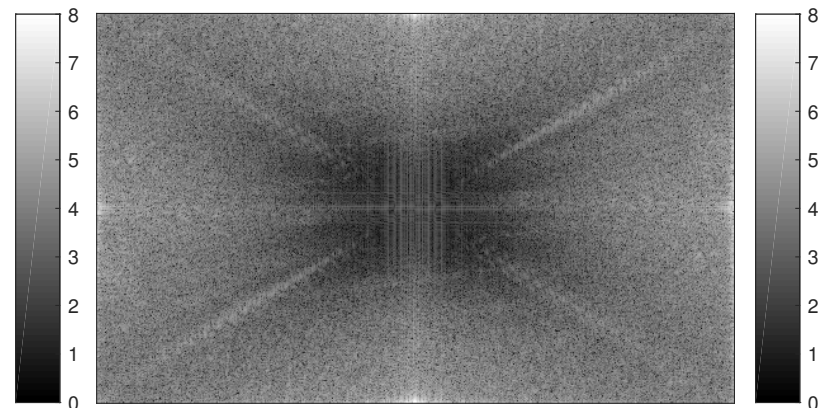

(d) $b i c-z 2(0.234,0.165)$

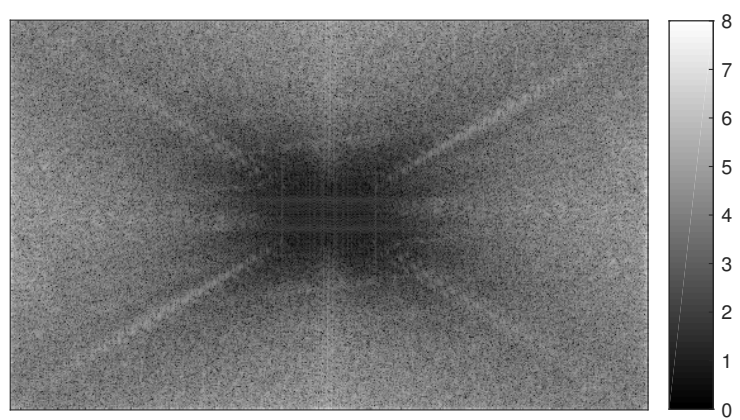

(f) $p+s$-bic $(0.172,0.146)$

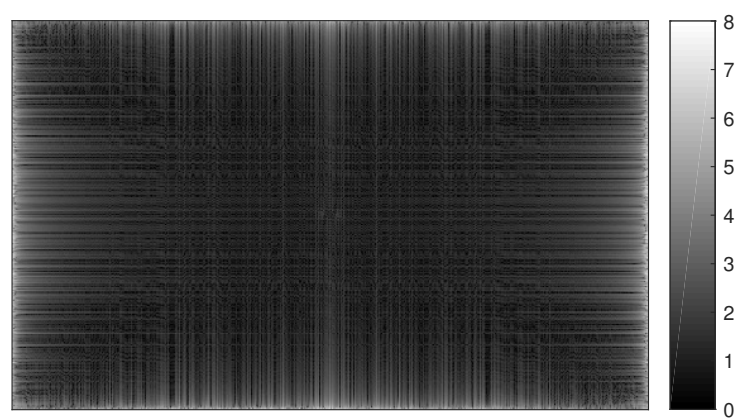

(h) $p+s$-tpi-spline1 $(0.0931,0.0372)$

Figure 4: Spectrum of the difference image $D I(\varphi)$ for various interpolation methods. The spectra correspond to the DFT in logarithmic scale $x \mapsto \log (1+x)$. The moderate homography $\varphi$ used is defined by the matrix $H_{\varphi}$ in $(23)$. The values between parentheses correspond to $\left(\mathcal{E}(\varphi), \mathcal{E}^{c}(\varphi)\right)$. For the bic, bic-z2 and $p+s$-bic methods i.e. the methods where the possibly zoomed images are interpolated by bicubic interpolation, the spectrum of the difference is high everywhere except in a small low-frequency region. For the spline11 method the corresponding region is larger. This explains why the structure of the image is visible in the difference image for these methods in Figure 3. For the tpi and spline11-z2 methods, the spectrum has non-negligible values in a band around the Nyquist frequency and in a vertical and horizontal cross structure. The cross structure is also visible in the bic-z2 method and is due to the incorrect handling of the non-periodicity. Indeed it disappears in the $p+s$-spline11-spline1 and $p+s$-tpi-spline1 methods, for which the error is mainly localized in a high-frequency band. 


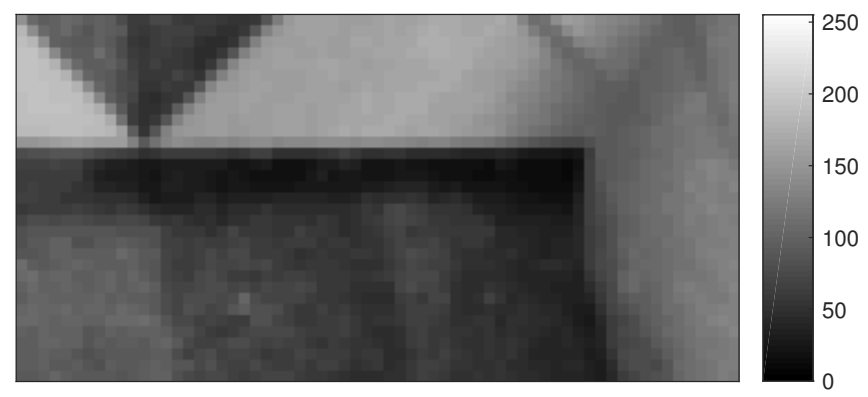

(a) Input

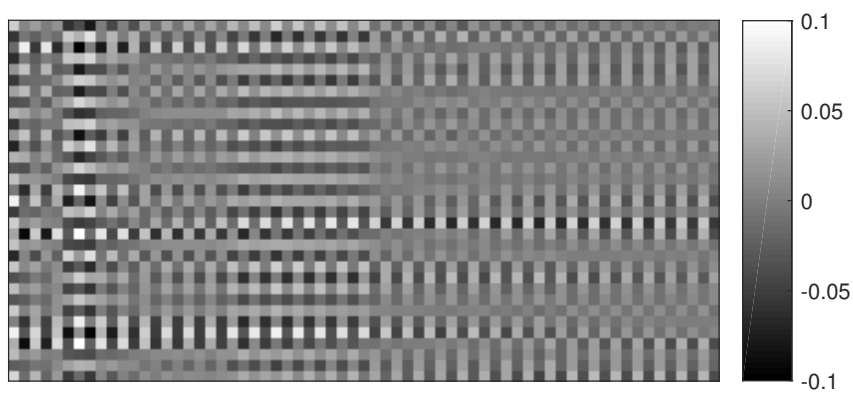

(b) $\operatorname{tpi}\left(\mathcal{E}^{c}(\varphi)=0.0877\right)$

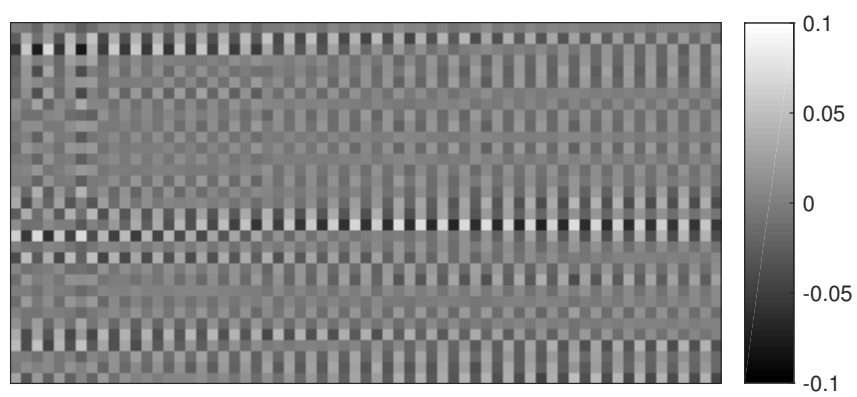

(d) $p+s$-spline11-spline1 $\left(\mathcal{E}^{c}(\varphi)=0.0372\right)$

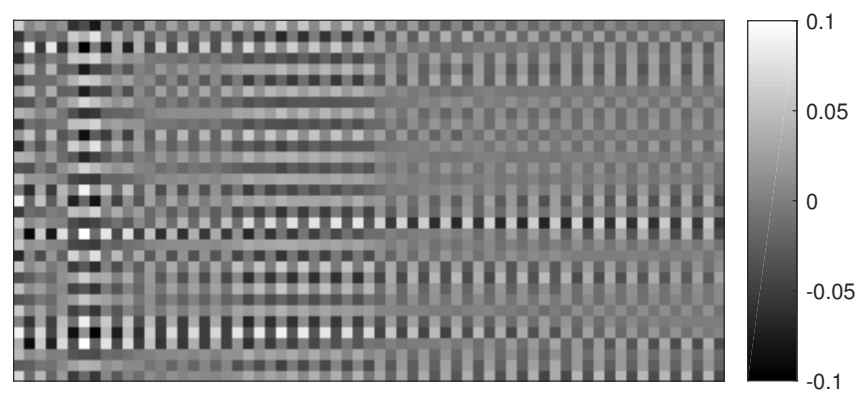

(c) spline11-z2 $\left(\mathcal{E}^{c}(\varphi)=0.0877\right)$

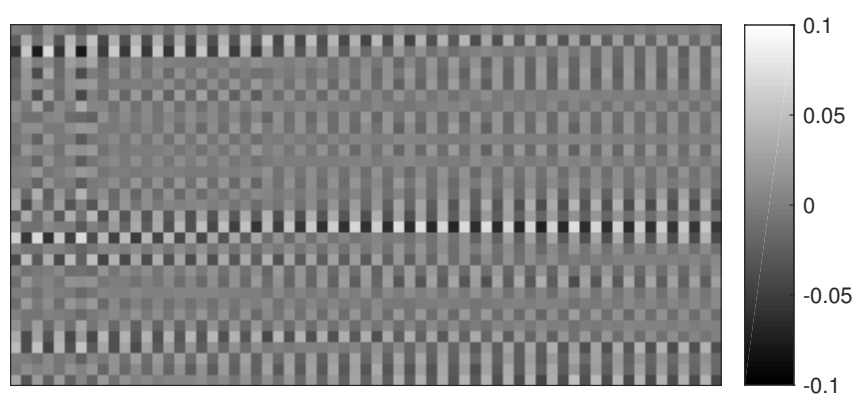

(e) $p+s$-tpi-spline1 $\left(\mathcal{E}^{c}(\varphi)=0.0372\right)$

Figure 5: Difference image $D I(\varphi)^{c}$ with spectrum clipping of ratio $r=5 \%$ for various interpolation methods. The moderate homography $\varphi$ used is defined by the matrix $H_{\varphi}$ in (23). The error is clearly lower when the periodic plus smooth decomposition is used. 

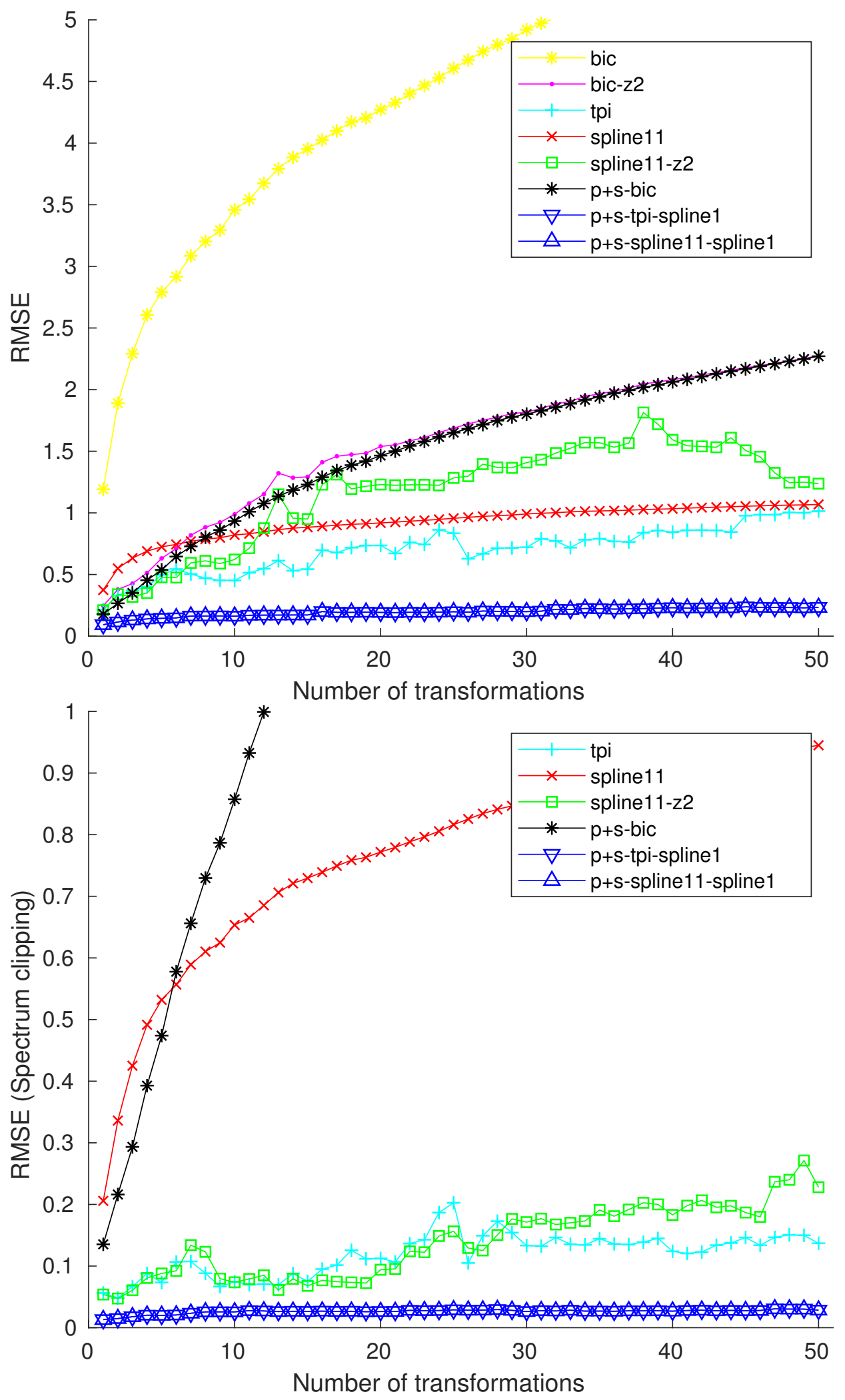

Figure 6: Evolution of the error after successive direct and inverse transformations for various interpolation methods (with half-symmetric extension). Up to $N_{\text {transf }}=50$ random moderate homographies are used. For the error with spectrum clipping (bottom), the bic and bic-z2 methods are not shown since not in the range. The curves of $p+s-t p i-s p l i n e 1$ and $p+s$-spline11-spline1 are indistinguishable since the results are almost the same. Globally the errors tend to increase with the number of transformations. The clipped versions of the error for the tpi, spline11-z2, $p+s-s p l i n e 11-s p l i n e 1$ and $p+s-$ tpi-spline1 methods are significantly smaller than the original error. We deduce that the error is mainly localized in a small high-frequency band. To prevent an important increase of the error $p+s$-spline11-*** or $p+s$-tpi-*** methods must be used. 


\section{Conclusion}

In this work, we introduced the reversibility error of interpolation methods. For a given image, it measures the error after applying successively a moderate homography, a crop (removing boundary artifacts) and the inverse homography. An average over random moderate homographies is made to remove the dependency on the homography. The clipped reversibility error is obtained by clipping the spectrum of the difference.

We also proposed new fine-tuned interpolation methods that are based on pre-existing interpolation methods. The zoomed version of an interpolation method is obtained by applying it to the DFT zoom-in of the image. It is closer to the trigonometric polynomial interpolation and has a better performance but it suffers from artifacts due to the non-periodicity of the image. In the periodic plus smooth version of interpolation methods, this non-periodicity is handled by applying the zoomed version to the periodic component and a base interpolation method, possibly of low order, to the smooth component.

In an experimental part, we have shown that the proposed fine-tuned methods have better reversibility errors than pre-existing interpolation methods and that the error is mainly localized in a small high-frequency band. The periodic plus smooth versions should be used to interpolate images. We recommend to use the periodic plus smooth versions of high order B-spline (e.g. p+s-spline11spline1). It is more efficient and provides better results than trigonometric polynomial interpolation.

\section{A Influence of the Image Content}

The conclusions of Section 5 on the order of the methods are clear-cut and do not depend on the choice of the image. However the interpolation methods provide in general worse results for images with a large amount of high-frequency content. Therefore the reversibility error is expected to increase with the amount of high-frequency content.

This statement is confirmed by the following experiment where we used the same setup as in Section 5.1 but with the Baboon image, which is the $512 \times 512$ color image shown in Figure 7 . Compared to the spectrum of the RubberWhale image (see Figure 2), its spectrum is flatter, which means that its energy is spread all over the frequencies. Indeed, $1 \%$ of the total energy is contained in the $1 \%$ highest frequencies while for the RubberWhale image this number was only of $0.2 \%$.

The results are presented in Table 2. The errors are higher than in Table 1 but the order of the interpolation methods and the conclusions remain the same.

\section{Acknowledgements}

The authors would like to thank Prof. Jean-Michel Morel for his support, suggestions, and many fruitful discussions.

Work partly financed by Office of Naval research grant N00014-17-1-2552, DGA Astrid project "filmer la Terre" no ANR-17-ASTR-0013-01, MENRT and Fondation Mathématique Jacques Hadamard.

\section{Image Credits}

Grayscale version of the Rubber Whale image from the Middlebury database [2]

Standard test image (Baboon image) 


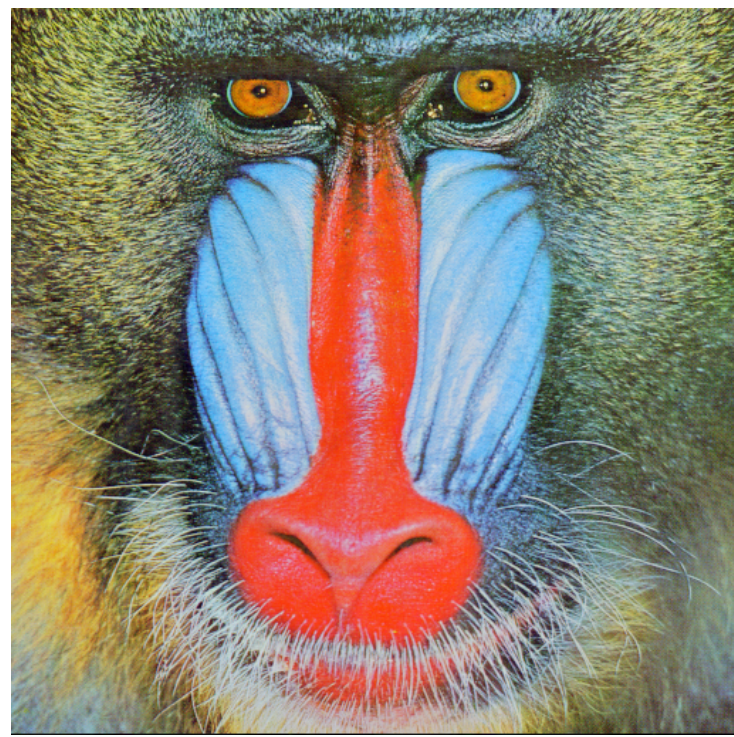

(a) Baboon image

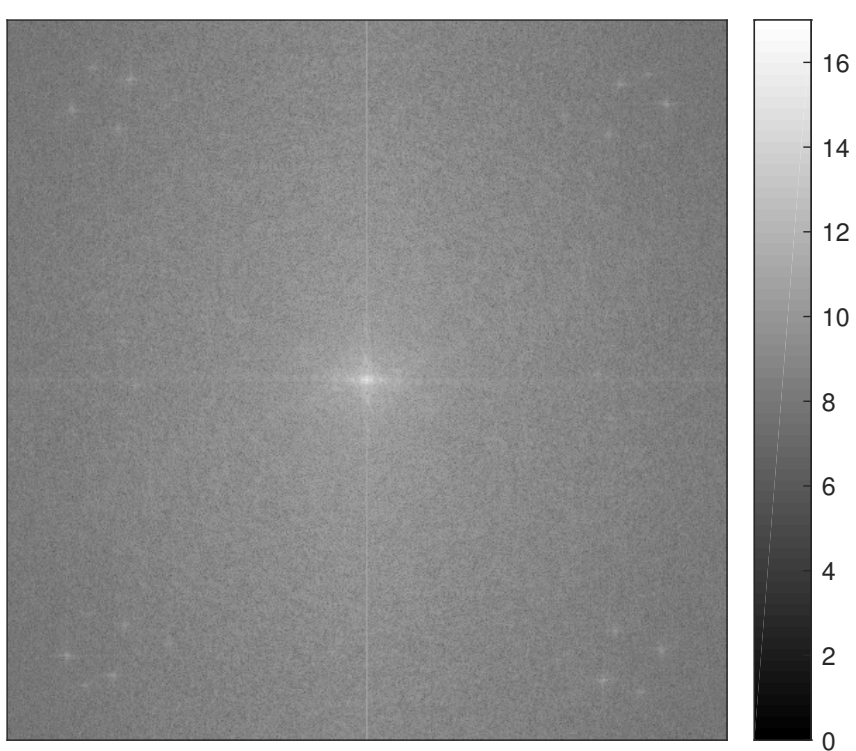

(b) Spectrum of (a)

Figure 7: Example of image with important high-frequency content. The spectrum in (b) corresponds to the discrete Fourier transform (RMS over the channels) in logarithmic scale $x \mapsto \log (1+x)$. The Baboon image in (a) is a $512 \times 512$ color image that contains a large amount of high-frequency content. Compared to the spectrum of the RubberWhale image (see Figure 2), the spectrum in (b) is flatter, which means that its energy is spread all over the frequencies. Indeed, $1 \%$ of the total energy is contained in the $1 \%$ highest frequencies while for the RubberWhale image this number was only of $0.2 \%$.

\begin{tabular}{l|l|l|l} 
& $\mathcal{E}$ & $\mathcal{E}^{c}$ & Time $(\mathrm{s})$ \\
\hline spline1 & 10.86765 & 10.76838 & 331 \\
bic & 6.81786 & 6.70694 & 402 \\
spline3 & 4.98228 & 4.83559 & 405 \\
spline11 & 2.52192 & 2.24597 & 1073 \\
tpi & 0.69723 & 0.24483 & 7436 \\
\hline spline1-z2 & 4.19696 & 4.08137 & 794 \\
bic-z2 & 1.22052 & 0.98217 & 842 \\
spline3-z2 & 0.73076 & 0.31325 & 947 \\
spline11-z2 & 0.69723 & 0.24483 & 1951 \\
\hline$p+$ s-spline1 & 4.19382 & 4.08060 & 993 \\
$p+$ s-bic & 1.20373 & 0.97732 & 1235 \\
p+s-spline3-spline1 & 0.69731 & 0.29490 & 1137 \\
$p+$-spline3 & 0.69731 & 0.29490 & 1237 \\
p+s-spline11-spline1 & $\mathbf{0 . 6 5 9 9 9}$ & $\mathbf{0 . 2 1 9 1 9}$ & $\mathbf{2 1 6 1}$ \\
p+s-spline11-spline3 & 0.65999 & 0.21919 & 2268 \\
$p+$-tpi-spline1 & 0.65999 & 0.21919 & 16858 \\
$p+$-tpi-spline3 & 0.65999 & 0.21919 & 16876
\end{tabular}

Table 2: Estimation of the reversibility errors for the Baboon image and $N_{\text {transf }}=1000$ random moderate homographies. The same experimental setup as in Section 5.1 was used. The errors are higher than in Table 1 but the order of the interpolation methods and the conclusions remain the same. 


\section{References}

[1] R. Abergel and L. Moisan, The Shannon Total Variation, Journal of Mathematical Imaging and Vision, (2017), pp. 1-30. http://dx.doi.org/10.1007/s10851-017-0733-5.

[2] S. Baker, D. Scharstein, J. P. Lewis, S. Roth, M. J. Black, and R. Szeliski, A Database and Evaluation Methodology for Optical Flow, International Journal of Computer Vision, 92 (2011), pp. 1-31. http://dx.doi.org/10.1007/s11263-010-0390-2.

[3] T. BRIAnd, Image Formation from a Large Sequence of RAW Images : performance and accuracy, PhD Thesis, Université Paris-Est, Nov. 2018. https://pastel.archives-ouvertes.fr/ tel-01980492.

[4] _ Trigonometric Polynomial Interpolation of Images, Image Processing On Line, 9 (2019), pp. 291-316. http://dx.doi.org/10.5201/ipol.2019.273.

[5] T. Briand And P. Monasse, Theory and Practice of Image B-Spline Interpolation, Image Processing On Line, 8 (2018), pp. 99-141. http://dx.doi.org/10.5201/ipol.2018.221.

[6] T. Briand, J. Vacher, B. Galerne, and J. Rabin, The Heeger \& Bergen Pyramid Based Texture Synthesis Algorithm, Image Processing On Line, 4 (2014), pp. 276-299. http://dx. doi.org/10.5201/ipol.2014.79.

[7] B. Galerne, Y. Gousseau, and J-M. Morel, Random Phase Textures: Theory and Synthesis, IEEE Transactions on Image Processing, 20 (2011), pp. 257-267. http://dx.doi.org/ 10.1109/TIP.2010.2052822.

[8] P. Getreuer, Linear Methods for Image Interpolation, Image Processing On Line, 1 (2011). http://dx.doi.org/10.5201/ipol.2011.g_lmii.

[9] T. Lin and J.L. Barron, Image Reconstruction Error for Optical Flow, Research in Computer and Robot Vision, (1995), pp. 269-290. http://dx.doi.org/10.1142/9789812812483_0016.

[10] L. Moisan, Periodic plus smooth image decomposition, Journal of Mathematical Imaging and Vision, 39 (2011), pp. 161-179. http://dx.doi.org/10.1007/s10851-010-0227-1.

[11] D. Potts, G. Steidl, And M. Tasche, Fast Fourier Transforms for Nonequispaced Data: A Tutorial, Birkhäuser Boston, Boston, MA, 2001, pp. 247-270. http://dx.doi.org/10.1007/ 978-1-4612-0143-4_12.

[12] L. Simon And J-M. Morel, Influence of Unknown Exterior Samples on Interpolated Values for Band-limited Images, SIAM Journal on Imaging Sciences, 9 (2016), pp. 152-184. http: //dx.doi.org/10.1137/140978338.

[13] P. Thévenaz, T. Blu, And M. Unser, Interpolation Revisited [Medical Images Application], IEEE Transactions on Medical Imaging, 19 (2000), pp. 739-758. http://dx.doi.org/10.1109/ 42.875199 . 